\title{
Application of Matlab/Simulink in Teaching Course of Communication Principle
}

\author{
Jing Chang, Xiaohui Li \\ Key Laboratory of Intelligent Computing \& Singnal Processing, Ministry of Education, School of Electronic Information \\ Engineering, Anhui University, Anhui Province, China \\ changing_2001@163.com,xhli@ahu.edu.cn
}

\begin{abstract}
This paper applies Matlab software as an auxiliary method in teaching course of communication principle. Considering variety of abstract concepts in this course, such as digital modulation and demodulation, channel-coding, random signal analysis, we select Matlab aid to provide dynamical simulation for communication theory. This method is used both in class presentation and laboratory experiments, which is helpful for students to understand the theory knowledge, enhancing their practical skills. Moreover, it can also extend students' ability of programming. Compared to traditional teaching method, the teacher's interpretation written on blackboard is the main means, this method enables students to study actively and cultivate their scientific qualities.
\end{abstract}

Index Terms - Matlab/Simulink, Communication Principle, auxiliary teaching method, curriculum experiments

\section{Introduction}

Communication principle is a course with abstract conceptions and complex mathematical deductions, which is complicated and unintelligible for students to learn. It is a core course in Communication Engineering Specialty. The contents include Signals and Systems, information theory and coding as well as probability and statistics. The key to learn this course well is to master the basic model and the principles of the whole communication system. Generally speaking, different communication system has the same architectural feature but with different model of function components. For example, as for analog communication, the transmitting equipment is the modulator, but as for digital communication system, the source can be transmitted reliably after source coding and channel coding, and the communication system structure always contain six parts, signal source, transmitting equipment, channel, receiving equipment, receiver, and noise. It is difficult for student to master complicated principles in very limited class hours. Students who want to learn it well should make combination with relative courses, such as signal processing, digital circuit, Probability theory. Also it requires the student to measure the quality of a communication system effectively. While the teachers who want to explain the academic theory about this course clearly should create various measures rather than monotonous interpreting on platform. It is necessary for teachers to explore new method to trigger students' interest and creativity on this course. To overcome those dilemma, aided teaching platform based on software by some scholars in universities have been proposed [1]-[3]. In [1], it is shown that teachers can play some demonstrative experiments in classroom or laboratory with the help of SystemVue, a software which can stimulate communication processing dynamically with visualized interface. Approaches that utilize Labview software have been pursued in [2] and [3]. Roughly speaking, Labview has the same function as SystemVue, which has modular unit and rich function library that is easy to use. But the above softwares lack the ability to train students programming capacity, which is significant in their postgraduate education. Given the insufficient aspects of above software we may utilize Matlab, which is widely used in scientific research and other industries, as a simulation platform to simulate communication processing [4][5]. Apart from being able to simulate communication system dynamically based on Simulink in Matlab, it is also possible to perform simulation by $\mathrm{m}$ file, which compiled in Matlab interface. $M$ file programming demands more mathematical reasoning in view of system function. With the help of Matlab, teachers can operate simulation test targeted contents being studied in class. making complex knowledge easy to master for students, improving their learning interest and encouraging them to practice by themselves. In this paper, our focus will be on the application of Matlab/Simulink in class to improve the effects and quality of communication principle teaching.

\section{The Procedure of Matlab/Simulink Simulation in Communication Principle}

The simulation based on Simulink can describe the whole process of communication vividly by providing system structure and signal waveform figure in every stage. You can follow these recommendations when using Simulink for test. First, confirming the category and main components of transmitting system circuit according to communication principle and drawing flow diagram about related modules of the system. Second, choosing and linking visual figure symbols, which come from function library in the design windows of Simulink. Then adjusting parameters of circuit components and outputting signal waveform figure in specific position of system circuit. Likewise, when simulating based on Matlab, there are three steps as follows. First, analyzing communication model feature in detail, including modulation and demodulation principle and channel status information in transmission. Second, writing and programming $\mathrm{m}$ file following Matlab specification, and then setting the related parameters to draw the conclusion, just like it is done in Simulink. 


\section{Teaching Methods Aided by Matlab/Simulink}

It is difficult for students to understand the complex theories, such as sampling theory, non-ISI transmission waveform, M-ary digital modulation and demodulation principle and complex digital modulation (such as MSK, QAM etc.), when teacher interprets these in platform in class. Also the students cannot master these principles through experiment boxes, which produced by different manufacturers, providing some simple basic experimental projects. But complex experiments cannot be completed by using experiment box only. With the aid of Matlab simulation software, these problems can be easily solved. Matlab simulation platform can make the abstract principles visualization, and the simulation based on $\mathrm{m}$ file is programmed by the principles of the algorithm from the core. But programming based on Matlab demands higher requirements for the theory level of learners. It is known to us that Simulink is produced on the basis of communication system structure, which is very suitable for the beginners of the major. Teachers can show students the simulation process in the classroom directly, helping them enhance the understanding of the relevant knowledge. Meanwhile, it is possible to teach them the programming methods, which is significant for them in their postgraduate study in the near future.

\section{A. low-pass sampling theory analysis based on Simulink}

It is known that the majority of the analog signal is low-pass signal (signal bandwidth is larger than the signal low frequency rate value), such as voice signal which frequency range from $300 \mathrm{~Hz}$ to $3.4 \mathrm{kHz}$ and it bandwidth is $3.1 \mathrm{kHz}$. These signal sampling should meet the low-pass sampling theory. The simulation about low-pass sampling theory is selected based on Simulink as an example.

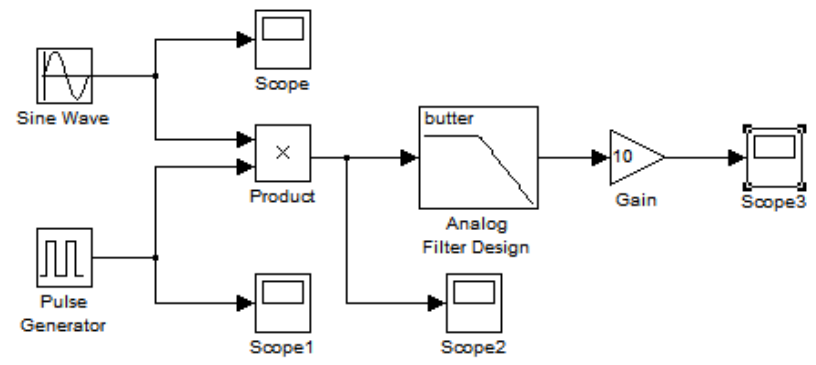

Fig.1 Block diagram of low-pass sampling circuit

Fig.1illustrates the key components of sampling circuit, such as a sine wave generator, a pulse generator, four scopes, a product, a low pass filter and an amplifier for connection to the sampling circuit. Suppose we would like to sample a signal $x(t)$, whose bandwidth is limited from 0 to $f_{H}$. If satisfied low-pass sampling theory, the sampling rate $f_{S}$ can be expressed as

$$
f_{S} \geq 2 f_{H}
$$

The original signal can be reconstructed without distortion from the sampled sequence. To make it easier, we take sine wave as the original signal, and its frequency is $10 \mathrm{~Hz}$. The process of sample is completed by the multiplier. We can get the original signal reconstructed from the sampled sequence using an analog low-pass filter, whose cut-off frequency is $12 \mathrm{~Hz}$. Changing the frequency of impulse sequence to test and verify low-pass sampling theory; the results are shown in Fig.2 a) to h).

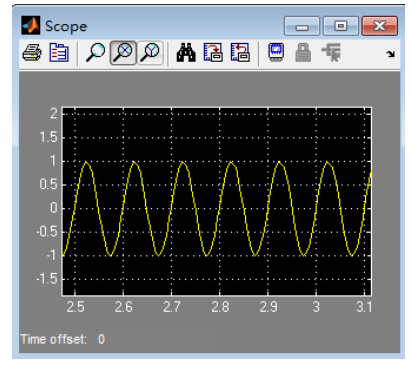

a) Original signal

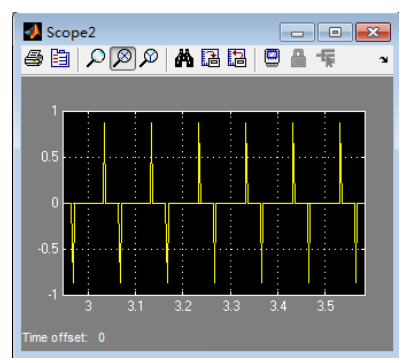

c) Sampled sequence

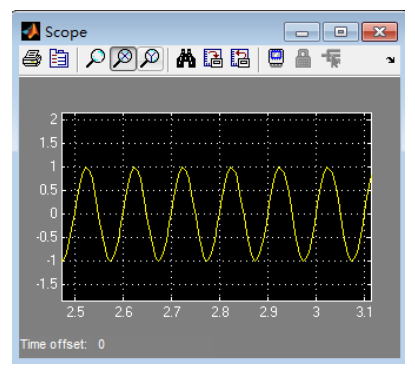

e) Original signal

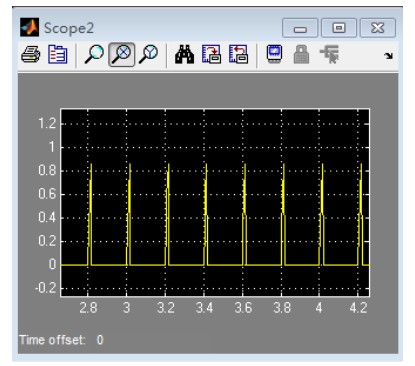

g) Sampled sequence

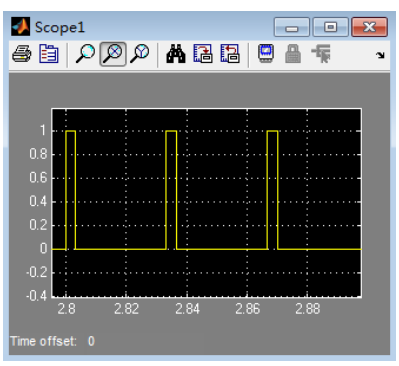

b) Impulse sequence $\left(f_{S}=30 H z\right)$

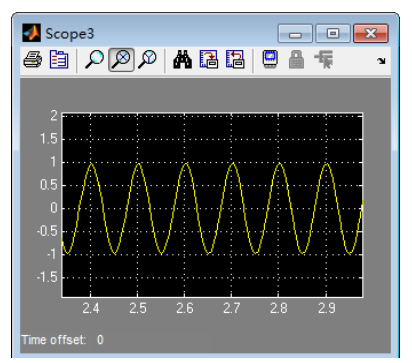

d) Reconstructed signal

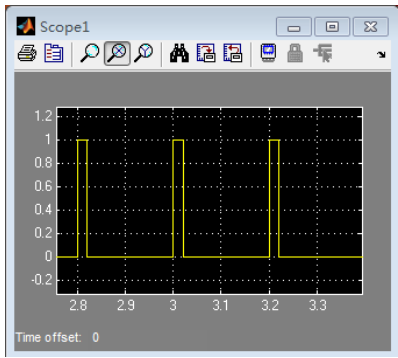

f) Impulse sequence $\left(f_{S}=5 \mathrm{~Hz}\right)$

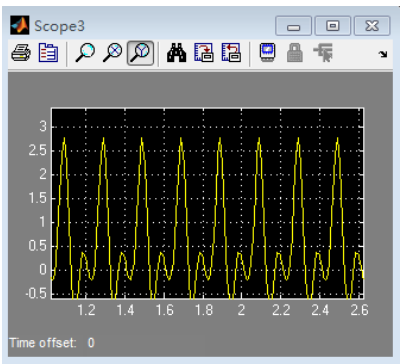

h) Reconstructed signal
Fig.2 simulation about low-pass sampling theory

Two examples of reconstructed signal are described in Figure3 d) and h), that is, when meets low-pass sampling 
theory $\left(f_{S} \geq 2 f_{H}\right)$ we can restore original signal without distortion, otherwise the reconstructed signal are distorted.

\section{B. Curriculum experiments of communication principle using $m$ file in Matlab}

In order to cultivate outstanding application-oriented engineer, a series of curriculum design experiments as course projects are developed for students. These experiments are to deal with problems in principle comprehension and programming. They are encouraged to consult extensive information in library, and discussion with their schoolmates or teachers. Students can perform their course projects on the simulation platform based on Matlab/Simulink, Systemview etc. Now we take the design of QPSK communication system as an example to interpret the application of Matlab. QPSK is also known as the four phase shift keying modulation, which is widely used in digital communication. In QPSK, each symbol contains 2 bit. There are four phase to map four different symbols in QPSK modulation. In this section, a typical QPSK transmission using quadrature modulation scheme is presented. QPSK modulation at transmitter can be described as converting the serial data stream into two parallel ones, then multiplying $\sin w t$ and $\cos w t$ to two parallel data stream respectively. Transmitting the modulated signal in additive white Gauss channel, and demodulating the signal at receiver. Further analysis of the BER curve of QPSK communication, based on the simulation of $\mathrm{m}$ files, is given in Fig.3.

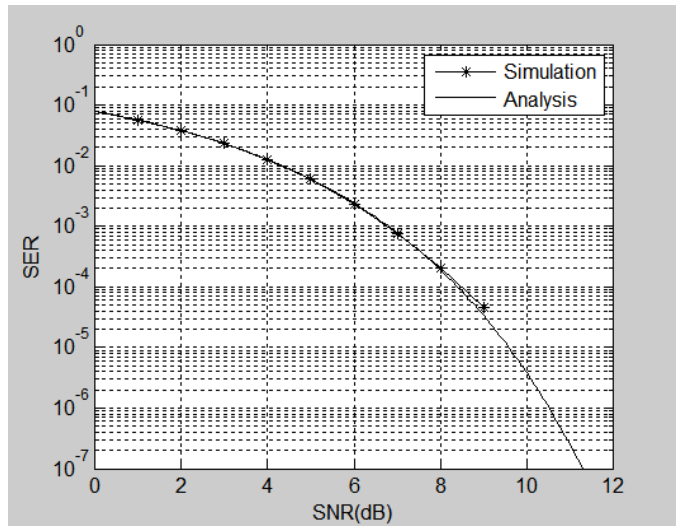

Fig.3 analytical and simulated SER in QPSK modulation

Fig.3 show the average SER versus average SNR per QPSK symbol in quadrature modulation scheme. The plot show curve generated through both analysis and simulation. The average analytical BER can be expressed as

$$
P_{S}=\frac{1}{2} \operatorname{erfc}(\sqrt{S N R})
$$

The simulated result is obtained after coherent demodulation. We see from Fig.3 that plot obtained through simulation are in good with the analytical one. We also see an improvement in interference rejection with increasing of channel condition (SNR). In the help of Matlab/Simulink, the student can master the sophisticated principles easily. Thus the students' abilities are improved, both practically and theoretically. Moreover, they can fully understand the core points of communication principle, such as signal coding and decoding, expression of channel condition, etc. We can also develop comprehensive experiments which reflect the new technology and the new development of communication based on Matlab/Simulink platform. Moreover, these trainings can give the students more opportunities to do more applied experiments and explore their research potential, improving their professional skills.

\section{Conclusion}

The application of Matlab/Simulink platform in teaching course of communication principle is presented. We improve the existing teaching methods with aid of Matlab. The comprehensive and innovative experiments about sampling theory and QPSK are carried out as examples to show that this Matlab aid teaching method can enhance students' practical abilities and scientific consciousness. And it also motivate the students' interests on this course, optimizing the teaching quality and providing an idea for training the ability of programming for students.

\section{References}

[1] The teaching research of wireless communication theory simulation experiment base on SystemVue, Cheng Wenqing, China Educational Technology \& Equipment, 2012(13), pp.50-54.

[2] Apply of LabVIEW in Experimental Teaching of the Principle of Digital Communication, CHEN Lingling, YANG Ming, LIU Xinjie, JOURNAL OF JILININ STITUTE OF CHEMICAL TECHNOLOGY, 2012, 29(11), pp.87-91.

[3] Application of LabVIEW in Communication Principle Experiment Teaching, SHAO Fen, LI Suwen, JIANG Enhua, ZHANG Yong, Experiment Science and Technology, 2013, 11(3), pp.4-6.

[4] Teaching and Practice of Communication Principles Course by the Aid of MatLab/Simulink, GUO Changying, HU Nianying, Modern Computer, 2012(10), 31-33.

[5] Auxiliary Teaching of MATLAB in the Communication Theory Course, JI Juan-juan, ZHANG Lan-fang, LI Qiang, DING Lei, Journal of Anqing Teachers College, 2013,19(1), pp.109-112. 